\title{
Author Correction: Treatment in myositis
}

Chester V. Oddis and Rohit Aggarwal

Nature Reviews Rheumatology (2018) https://doi.org/10.1038/nrrheum.2018.42

Published online 29 March 2018.

In the originally published version of this article, several references in Table 1 were incorrect.

These errors have now been corrected in the HTML and PDF versions of the manuscript.

https://doi.org/10.1038/s41584-018-0086-5 I Published online 11 September 2018 